ISSN 0258-7122 (Print), 2408-8293 (Online)

Bangladesh J. Agril. Res. 44(4): 631-640, December 2019

\title{
MOISTURE LEVEL AND STORAGE CONTAINER EFFECTS ON SEED QUALITY OF SOYBEAN GENOTYPES UNDER AMBIENT CONDITION
}

\author{
M. R. ALI' ${ }^{1}$, M. M. RAHMAN ${ }^{2}$, M. ASADUZZAMAN ${ }^{3}$ \\ M. A. H. KHAN ${ }^{4}$ AND J. RAHMAN ${ }^{5}$
}

\begin{abstract}
The experiment was conducted at the Seed Laboratory, Regional Agricultural Research Station, Bangladesh Agricultural Research Institute, Jamalpur in 2013 to study the effect of seed moisture content and storage containers on seed quality of soybean genotypes. Three genotypes of soybean (AGS 191, ASET 93 and Shohag), four initial seed moisture content $(6,8,10$ and 12\%) and two types of storage containers (polythene bag and glass jar) were included in the experimental treatment. Seeds of soybean genotypes was stored at ambient condition were temperature ranged from 15.97 to $29.37{ }^{\circ} \mathrm{C}$, relative humidity ranged from 75.21 to $86.23 \%$ and rainfall ranged from 0.00 to $425 \mathrm{~mm}$ during the whole storage period. Seed moisture content (\%), germination (\%) and vigour index were recorded at 50,100, 150 and 200 days after storage (DAS). Result showed that final seed moisture content increased with the increase of initial seed moisture content. Genotype AGS 191 showed the highest germination $(\%)$ and vigour index. Seeds stored in polythene bag or glass jar showed similar performance for germination (\%) and vigour index. Highest seed moisture content significantly reduced the germination and vigour index errespective of containers. The results indicate that soybean seed can be stored safely for at least 200 days maintaining $>80 \%$ germination and high vigour when stored in polythene bag or glass jar with 6-8\% initial moisture content at ambient room temperature and relative humidity.
\end{abstract}

Keywords: Soybean, seed moisture, storage container, vigour

\section{Introduction}

Soybean (Glycine max [L.] Merrill) is the world's most important grain legume crop in terms of total production and international trade. Soybean seed contains $36-47 \%$ protein and $15-25 \%$ oil, $20-26 \%$ carbohydrate (Rahman, 2001). It is a high and available source of calcium, phosphorus, iron, thiamine including vitamins $\mathrm{A}, \mathrm{B}, \mathrm{C}$ and $\mathrm{D}$. The common people of Bangladesh can not afford animal protein like egg, milk, meat and fish in their daily diet because of their high cost. The demand of soybean is increasing for making different food

${ }^{1}$ Principal Scientific Officer, RARS, Jamalpur, ${ }^{2}$ Professor, Department of Agronomy, Bangladesh Agricultural University (BAU), Mymensingh, ${ }^{3}$ Senior Scientific Officer, RARS, Jamalpur, ${ }^{4}$ Senior Scientific Officer, OFRD, Mymensingh, ${ }^{5}$ Scientific Officer, RARS, Jamalpur, Bangladesh. 
products for human consumption as well as feed for poultry and fish in Bangladesh. The soil and climatic condition of Bangladesh is congenial for soybean production. The lack of supply of quality seed to the farmers render major problem in the expansion of area under soybean cultivation. It has been reported that soybean seed is short lived and loses its viability in very short period of storage even with good nonporous storage container. Arunnandhy and Senanayaka (1991) reported that the soybean seeds can be kept with high germination and vigour for more than one year when stored in sealed containers. Storage containers or packaging materials are considered as one of the most important factors influencing longevity of seeds in storage. Storage container had significant effect on moisture content of wheat seed at different observation date of storing (Rahman et al., 2010). When seed could be packaged in moisture proof containers, the relative humidity of the air around the seed remains low, then the seed equilibrium moisture remains low and the seed maintains its viability and vigour for a longer time (Poonam et al., 2001; Agha et al., 2004). Storage container influences seed quality and initial adjustment of seed moisture played a vital role in the preservation and longevity of seeds. The physical properties and storage potential of seed were influenced to a very large extent by moisture content and the relative humidity of the atmosphere surrounding the seed (Kong et al., 2008). Reports are also available that polythene bags can be used as moisture proof container (Alam and Rahman, 2005). Soybean seeds stored in moisture proof containers retain high viability than those in moisture permeable containers (Tatipata, 2009). Depending on initial condition, seed deteriorates less rapidly in impermeable then permeable containers. Use of proper storage container to maintain the quality of farmer-saved seed in storage and preserving its viability should be an important consideration to reduce seed loss and increase crop yield. In view of the above facts, the present study was undertaken to develop appropriate storage techniques to maintain desired viability and vigour of soybean seeds.

\section{Materials and Methods}

The experiment was conducted at Seed Laboratory of Regional Agricultural Research Station, BARI, Jamalpur during May to December 2013. Daily temperature (maximum, minimum and mean), total monthly rainfall and monthly relative humidity were collected from records of the Regional Agricultural Research Station, Jamalpur. The average monthly maximum and minimum temperature, relative humidity and total rainfall that prevailed during the experimental period have been presented in Table 1. The maximum and minimum temperatures were found in the month of April and January, Heavy rainfall was observed during the month of April to October whereas it was scanty during the month of November to March. There was no rainfall in the month of December. Monthly average relative humidity ranged from 75.21 to $86.23 \%$. 
Table 1. Monthly record of air temperature, rainfall and relative humidity the experimentation period at Bangladesh Agricultural Research Station, Jamalpur from January to December 2013

\begin{tabular}{l|c|c|c|c|c}
\hline \multirow{2}{*}{ Month } & \multicolumn{3}{|c|}{ Air temperature } & Relative humidity & $\begin{array}{c}\text { Rain fall } \\
(\%)\end{array}$ \\
\cline { 2 - 4 } & Maximum & Minimum & Average & 83.65 & Trace \\
\hline January & 21.77 & 10.17 & 15.97 & 75.21 & 27.10 \\
February & 26.67 & 15.49 & 21.08 & 75.39 & 114.00 \\
March & 27.95 & 18.11 & 23.03 & 80.07 & 96.20 \\
April & 31.63 & 22.25 & 26.94 & 81.13 & 265.00 \\
May & 32.03 & 23.33 & 27.68 & 86.23 & 425.30 \\
June & 30.57 & 25.68 & 28.13 & 85.00 & 212.90 \\
July & 31.81 & 26.89 & 29.35 & 85.00 & 192.40 \\
August & 31.36 & 26.42 & 28.89 & 84.23 & 330.90 \\
September & 32.22 & 25.95 & 29.09 & 84.77 & 105.80 \\
October & 31.29 & 22.89 & 27.09 & 82.53 & 83.10 \\
November & 28.79 & 18.54 & 23.67 & 84.06 & 00.00 \\
December & 25.32 & 14.40 & 19.86 & &
\end{tabular}

Source: Regional Agricultural Research Station, Jamalpur.

Three soybean genotypes (AGS 191, ASET 93 and Shohag), two storage container (polythene bag and glass jar) and four level of initial seed moisture content $(6,8,10$ and $12 \%)$ were used as treatment variables. The experiment was laid out in a Completely Randomized Design with three replications. The crop was grown at Regional Agricultural Research Station Farm, Jamalpur with proper agronomic management. The crop was harvested at full maturity and after proper processing, cleaning and drying the seed was stored in polythene bags until used for experimentation. The seed was dried in the sun on a tripale set on the cemented floor to about $6 \%$ initial seed moisture content (SMC). Just before final storage the seed was re-hydrated at $70 \%$ relative humidity $(\mathrm{RH})$ for required period of time to obtain targeted moisture contents were achieved. Each container was completely filled with seed as per experimental specification and then made air tight. The seed was tested for different quality parameters at 50,100,150 and 200 days after storage (DAS). The containers were kept in the laboratory under ambient room condition $\left(23-32^{\circ} \mathrm{C}\right.$ temperature and $78.64 \%$ relative humidity). The quality parameters tested were seed moisture content, germination percentage and vigour index.

Seed moisture content was measured using high constant temperature oven dry method following ISTA rules (2003). About 5-8g of seeds were taken in the 
alluminium dish and dried in the oven at $130{ }^{\circ} \mathrm{C}$ for 2 hours (until constant weight reached). Germination test was done in sand culture method. Two third of a plastic dish $(20 \mathrm{~cm}$ diameter and $15 \mathrm{~cm}$ deep) was filled with sterilized sand having $60 \%$ water holding capacity. Randomly collected 100 seeds from each container were placed into the sand for the germination test. The germination dishes were placed in the germination cabinet and seedling evaluation was done at 8 days after placing the test. The number of normal seedlings per dish was regarded as the germination percentage. Germination index of seed was estimated from the seed set in the germination test by calculating the vigour index formula. The seedling emerged each day having radical length of $2 \mathrm{~cm}$ or more was considered as germinated. At the end of the storage period, randomly selected 100 seeds from each seed lot in three replications were sown in the well prepared field. The number of seedlings emerged each day were counted up to 15 days after sowing.

Data analysis was done following the analysis of variance (ANOVA) technique and mean differences were adjusted by Duncan's New Multiple Range Test at $5 \%$ level of significance with a computer package programme MSTAT-C.

\section{Results and Discussion}

\section{Seed moisture content (SMC)}

The effect of storage container, initial seed moisture content and genotypes on seed moisture content and germination performance was evaluated in terms of germination, germination index and field performance. The interaction effect of storage container, initial seed moisture content and genotypes on moisture content of soybean seed was statistically significant at each of the observation dates during the storage period in 2013. The moisture content of ASET 93 stored in glass jar with 6\% initial MC were $6.90,7.23,8.09$ and $8.70 \%$, respectively at $50,100,150$ and 200 DAS and it was statistically at par to those of AGS 191 or ASET 93 or Shohag in polythene bag or glass jar with 6\% initial MC with, respectively (Table 1). The moisture content of Shohag stored in polythene bag at $12 \%$ initial MC were $12.40,12.70,13.40$ and $13.93 \%$, respectively at 50 , 100, 150 and 200 DAS and it was statistically at par to those of AGS 191 and ASET 93 stored in glass jar or polythene bag with 12\% initial MC (Table 2). When seed could be packaged in moisture proof containers, the relative humidity of the air around the seed remains low, then the seed equilibrium moisture remains low (Poonam et al., 2001; Agha et al., 2004, Kong et al., 2008). Similar genetic variations in moisture content of soybean seed have also been reported by Tatic et al., 2012. These results are in good agreement with those reported by Tatipata (2009). 
Table 2. Interaction effect of storage container, genotype and initial seed moisture content onmoisture content of soybean seed at different days after storage

\begin{tabular}{|c|c|c|c|c|}
\hline \multirow{2}{*}{$\begin{array}{l}\text { Container×genotype } \\
\times \text { initial SMC }\end{array}$} & \multicolumn{4}{|c|}{ Seed moisture content $(\%)$ at different days after storage } \\
\hline & 50 & 100 & 150 & 200 \\
\hline $\mathrm{C}_{1} \mathrm{~V}_{1} \mathrm{M}_{1}$ & $7.14 \mathrm{e}$ & $7.60 \mathrm{e}$ & 8.40def & $8.95 \mathrm{ef}$ \\
\hline $\mathrm{C}_{1} \mathrm{~V}_{1} \mathrm{M}_{2}$ & $8.36 \mathrm{~d}$ & $8.90 \mathrm{c}$ & $9.50 \mathrm{cb}$ & $10.03 \mathrm{~d}$ \\
\hline $\mathrm{C}_{1} \mathrm{~V}_{1} \mathrm{M}_{3}$ & $10.47 \mathrm{c}$ & $11.04 \mathrm{~b}$ & $11.60 \mathrm{~b}$ & $12.13 b$ \\
\hline $\mathrm{C}_{1} \mathrm{~V}_{1} \mathrm{M}_{4}$ & $12.35 \mathrm{a}$ & $12.60 \mathrm{a}$ & $13.25 \mathrm{a}$ & $13.64 \mathrm{a}$ \\
\hline $\mathrm{C}_{1} \mathrm{~V}_{2} \mathrm{M}_{1}$ & $7.07 \mathrm{e}$ & $7.55 \mathrm{e}$ & $8.32 \mathrm{ef}$ & $8.96 \mathrm{ef}$ \\
\hline $\mathrm{C}_{1} \mathrm{~V}_{2} \mathrm{M}_{2}$ & $8.50 \mathrm{~d}$ & $8.90 \mathrm{c}$ & $9.21 \mathrm{c}-\mathrm{f}$ & $10.10 \mathrm{~d}$ \\
\hline $\mathrm{C}_{1} \mathrm{~V}_{2} \mathrm{M}_{3}$ & $10.28 \mathrm{c}$ & $10.80 \mathrm{~b}$ & $11.40 \mathrm{~b}$ & $11.93 b c$ \\
\hline $\mathrm{C}_{1} \mathrm{~V}_{2} \mathrm{M}_{4}$ & $12.40 \mathrm{a}$ & $12.70 \mathrm{a}$ & $13.04 \mathrm{a}$ & $13.32 \mathrm{a}$ \\
\hline $\mathrm{C}_{1} \mathrm{~V}_{3} \mathrm{M}_{1}$ & $7.08 \mathrm{e}$ & 7.80de & $8.50 c-f$ & $9.02 \mathrm{ef}$ \\
\hline $\mathrm{C}_{1} \mathrm{~V}_{3} \mathrm{M}_{2}$ & $8.38 \mathrm{~d}$ & $8.96 \mathrm{c}$ & $9.53 \mathrm{c}$ & $10.16 \mathrm{~d}$ \\
\hline $\mathrm{C}_{1} \mathrm{~V}_{3} \mathrm{M}_{3}$ & $10.39 \mathrm{c}$ & $10.80 \mathrm{~b}$ & $11.43 b$ & $11.85 b c$ \\
\hline $\mathrm{C}_{1} \mathrm{~V}_{3} \mathrm{M}_{4}$ & $12.40 \mathrm{a}$ & $12.70 \mathrm{a}$ & $13.40 \mathrm{a}$ & $13.93 \mathrm{a}$ \\
\hline $\mathrm{C}_{2} \mathrm{~V}_{1} \mathrm{M}_{1}$ & $7.01 \mathrm{e}$ & $7.48 \mathrm{e}$ & $8.21 \mathrm{f}$ & $8.86 \mathrm{ef}$ \\
\hline $\mathrm{C}_{2} \mathrm{~V}_{1} \mathrm{M}_{2}$ & $8.44 \mathrm{~d}$ & $8.90 \mathrm{c}$ & $9.33 \mathrm{~cd}$ & $9.87 \mathrm{~d}$ \\
\hline $\mathrm{C}_{2} \mathrm{~V}_{1} \mathrm{M}_{3}$ & $11.02 \mathrm{~b}$ & $11.30 \mathrm{~b}$ & $11.65 \mathrm{~b}$ & $11.87 b c$ \\
\hline $\mathrm{C}_{2} \mathrm{~V}_{1} \mathrm{M}_{4}$ & $12.30 \mathrm{a}$ & $12.70 \mathrm{a}$ & $13.20 \mathrm{a}$ & $13.49 \mathrm{a}$ \\
\hline $\mathrm{C}_{2} \mathrm{~V}_{2} \mathrm{M}_{1}$ & $6.90 \mathrm{e}$ & $7.29 \mathrm{e}$ & $8.09 \mathrm{f}$ & $8.70 \mathrm{f}$ \\
\hline $\mathrm{C}_{2} \mathrm{~V}_{2} \mathrm{M}_{2}$ & $8.43 \mathrm{~d}$ & $8.70 \mathrm{c}$ & $9.15 c-f$ & $9.49 \mathrm{de}$ \\
\hline $\mathrm{C}_{2} \mathrm{~V}_{2} \mathrm{M}_{3}$ & $10.21 \mathrm{c}$ & $10.60 \mathrm{~b}$ & $11.02 \mathrm{~b}$ & $11.36 \mathrm{c}$ \\
\hline $\mathrm{C}_{2} \mathrm{~V}_{2} \mathrm{M}_{4}$ & $12.31 \mathrm{a}$ & $12.60 \mathrm{a}$ & $13.02 \mathrm{a}$ & $13.30 \mathrm{a}$ \\
\hline $\mathrm{C}_{2} \mathrm{~V}_{3} \mathrm{M}_{1}$ & $7.00 \mathrm{e}$ & $7.40 \mathrm{e}$ & $8.10 \mathrm{f}$ & $8.73 f$ \\
\hline $\mathrm{C}_{2} \mathrm{~V}_{3} \mathrm{M}_{2}$ & $8.22 \mathrm{~d}$ & $8.60 \mathrm{~cd}$ & $9.10 \mathrm{c}-\mathrm{f}$ & $9.50 \mathrm{de}$ \\
\hline $\mathrm{C}_{2} \mathrm{~V}_{3} \mathrm{M}_{3}$ & $10.20 \mathrm{c}$ & $10.60 \mathrm{~b}$ & $11.00 \mathrm{~b}$ & $11.40 \mathrm{c}$ \\
\hline $\mathrm{C}_{2} \mathrm{~V}_{3} \mathrm{M}_{4}$ & $12.20 \mathrm{a}$ & $12.70 \mathrm{a}$ & $13.19 \mathrm{a}$ & $13.53 \mathrm{a}$ \\
\hline $\mathrm{CV}(\%)$ & 4.28 & 6.29 & 5.34 & 7.21 \\
\hline
\end{tabular}

$\mathrm{CV}=$ Coefficient of variation, $\mathrm{C}_{1}=$ Polythene bag, $\mathrm{C}_{2}=$, Glass jar, $\mathrm{V}_{1}=\mathrm{AGS}-191, \mathrm{~V}_{2}=$ ASET-93, $\mathrm{V}_{3}=$ Shohag, $\mathrm{SMC}=$ Seed moisture content, $\mathrm{M}_{1}=6 \% \mathrm{SMC}, \mathrm{M}_{2}=8 \% \mathrm{SMC}$, $\mathrm{M}_{3}=10 \% \mathrm{SMC}, \mathrm{M}_{4}=12 \% \mathrm{SMC}$, In a column, figures having similar letter(s) do not differ significantly by DMRT. 


\section{Germination percentage}

The interaction of effect of storage container, genotypes and initial seed moisture content on germination percentage was statistically significant at each of the observation dates during the storage period in 2013. The germination percentage of AGS 191 stored in glass jar with 6\% MC were 96.66, 94.00, 93.33 and $92.00 \%$, respectively at 50,100,150 and 200 DAS and it was statistically at par to those of AGS 191 or ASET 93 or Shohag stored in glass jar or polythene bag with at $6 \%$ and $8 \%$ initial SMC. Those values for Shohag in polythene bag with $12 \%$ initial SMC were 74.00, 62.00, 24.00 and 6.33\%, respectively (Table 3). These results are conformity of those reported by Balesevic-Tubic et al., 2005. Storage containers or packaging materials are considered as one of the most important factors influencing longevity of seeds in storage. Storage container had significant effect on moisture content of wheat seed at different observation date of storing (Rahman et al., 2010 and Ali et al., 2018).

\section{Field emergence performance}

Field emergence performance was evaluated in terms of field emergence and mean germination time in field. Soybean genotype, storage container and initial seed moisture content had significant effect on field emergence of soybean seed at 200 DAS (Table 3). The field emergence percentage of AGS 191 seed stored in polythene bag were $88.66,88.00,36.00$ and $8.00 \%$ at $6,8,10$ and $12 \%$ initial SMC while those stored in glass jar were 90.00, 88.00, 54.00 and $16.00 \%$, respectively (Table 3). The field emergence of ASET 93 seed stored in polythene bag were $86.30,83.33,42.66$ and $6.00 \%$ at $6,8,10$ and $12 \%$ initial SMC while those stored in glass jar were $86.00,84.00,40.00$ and $18.00 \%$, respectively (Table 3). The field emergence of Shohag seed stored in polythene bag were $80.00,80.00,16.33$ and $3.33 \%$ at $6,8,10$ and $12 \%$ initial SMC while those stored in glass jar were $80.00,80.00,36.00$ and $12.00 \%$, respectively (Table 3 ). Though the field emergence (\%) among the genotype was statistically identical but AGS 191 showed the best performance followed by ASET 93 and Shohag. Upto $8 \%$ SMC, more than $80 \%$ field emergence was observed. After that the same was drastically declined irrespective of container. Poonam et al. (2001) also reported that seeds of soybean cv. PK-327 at moisture content of 7-8\% stored in single and double polyethylene bags could be stored for up to 8 months under ambient conditions. 
Table 3. Interaction effect of storage container, genotype and initial seed moisture content on germination and field emergence of soybean seed

\begin{tabular}{|c|c|c|c|c|c|}
\hline \multirow{2}{*}{$\begin{array}{c}\text { Container×genotypex } \\
\text { initial SMC }\end{array}$} & \multicolumn{4}{|c|}{ Germination $(\%)$ at different days after storage } & \multirow{2}{*}{$\begin{array}{c}\text { Field emergence } \\
\text { at } 200 \text { days after } \\
\text { storage }\end{array}$} \\
\hline & 50 & 100 & 150 & 200 & \\
\hline $\mathrm{C}_{1} \mathrm{~V}_{1} \mathrm{M}_{1}$ & $96.33 \mathrm{a}$ & $94.00 \mathrm{a}$ & $92.00 \mathrm{a}$ & $91.66 \mathrm{a}$ & $88.66 \mathrm{a}$ \\
\hline $\mathrm{C}_{1} \mathrm{~V}_{1} \mathrm{M}_{2}$ & $96.00 \mathrm{a}$ & $93.33 \mathrm{ab}$ & $90.92 \mathrm{ab}$ & $90.00 \mathrm{a}$ & $88.00 \mathrm{a}$ \\
\hline $\mathrm{C}_{1} \mathrm{~V}_{1} \mathrm{M}_{3}$ & $89.33 c-f$ & $83.33 \mathrm{de}$ & $62.00 \mathrm{e}$ & $40.00 \mathrm{ef}$ & $36.00 \mathrm{c}$ \\
\hline $\mathrm{C}_{1} \mathrm{~V}_{1} \mathrm{M}_{4}$ & $80.00 \mathrm{gh}$ & $60.00 \mathrm{~g}$ & 30.00hi & $8.66 \mathrm{i}$ & $8.00 \mathrm{def}$ \\
\hline $\mathrm{C}_{1} \mathrm{~V}_{2} \mathrm{M}_{1}$ & $94.00 \mathrm{ab}$ & $92.33 \mathrm{abc}$ & $88.66 \mathrm{abc}$ & $87.33 \mathrm{abc}$ & $86.3 \mathrm{a}$ \\
\hline $\mathrm{C}_{1} \mathrm{~V}_{2} \mathrm{M}_{2}$ & $94.00 \mathrm{abc}$ & $92.00 \mathrm{a}-\mathrm{d}$ & $88.00 \mathrm{abc}$ & $85.33 \mathrm{abc}$ & $83.33 \mathrm{a}$ \\
\hline $\mathrm{C}_{1} \mathrm{~V}_{2} \mathrm{M}_{3}$ & $84.33 \mathrm{efg}$ & $72.00 f g$ & $64.00 \mathrm{e}$ & $46.66 \mathrm{e}$ & $42.66 b c$ \\
\hline $\mathrm{C}_{1} \mathrm{~V}_{2} \mathrm{M}_{4}$ & $78.00 \mathrm{gh}$ & $60.00 \mathrm{~g}$ & 33.00ghi & $10.00 \mathrm{i}$ & $6.00 \mathrm{ef}$ \\
\hline $\mathrm{C}_{1} \mathrm{~V}_{3} \mathrm{M}_{1}$ & $92.00 \mathrm{a}-\mathrm{d}$ & $86.00 \mathrm{~b}-\mathrm{e}$ & $84.00 b c$ & $82.00 b c$ & $80.00 \mathrm{a}$ \\
\hline $\mathrm{C}_{1} \mathrm{~V}_{3} \mathrm{M}_{2}$ & $92.00 \mathrm{a}-\mathrm{d}$ & $84.00 \mathrm{cde}$ & $82.00 \mathrm{~cd}$ & $80.00 \mathrm{c}$ & $80.00 \mathrm{a}$ \\
\hline $\mathrm{C}_{1} \mathrm{~V}_{3} \mathrm{M}_{3}$ & $89.33 c-f$ & $72.00 \mathrm{~g}$ & $43.33 \mathrm{fg}$ & $21.33 \mathrm{~h}$ & $16.33 d$ \\
\hline $\mathrm{C}_{1} \mathrm{~V}_{3} \mathrm{M}_{4}$ & $74.00 \mathrm{~h}$ & $62.00 \mathrm{~g}$ & $24.00 \mathrm{i}$ & $6.33 \mathrm{i}$ & $3.33 \mathrm{f}$ \\
\hline $\mathrm{C}_{2} \mathrm{~V}_{1} \mathrm{M}_{1}$ & $96.66 \mathrm{a}$ & $94.00 \mathrm{a}$ & $93.33 \mathrm{a}$ & $92.00 \mathrm{a}$ & $90.00 \mathrm{a}$ \\
\hline $\mathrm{C}_{2} \mathrm{~V}_{1} \mathrm{M}_{2}$ & $96.00 \mathrm{ab}$ & $93.66 \mathrm{ab}$ & $92.33 \mathrm{a}$ & $91.33 \mathrm{a}$ & $90.00 \mathrm{a}$ \\
\hline $\mathrm{C}_{2} \mathrm{~V}_{1} \mathrm{M}_{3}$ & $90.00 c-f$ & $84.00 \mathrm{cde}$ & $74.00 \mathrm{~d}$ & $62.00 \mathrm{~d}$ & $54.00 \mathrm{~b}$ \\
\hline $\mathrm{C}_{2} \mathrm{~V}_{1} \mathrm{M}_{4}$ & 82.00fgh & $62.00 \mathrm{~g}$ & $42.00 \mathrm{fg}$ & $27.00 \mathrm{gh}$ & $16.00 \mathrm{~d}$ \\
\hline $\mathrm{C}_{2} \mathrm{~V}_{2} \mathrm{M}_{1}$ & $94.00 \mathrm{abc}$ & $92.00 \mathrm{abc}$ & $90.00 \mathrm{ab}$ & 88.00ab & $86.00 \mathrm{a}$ \\
\hline $\mathrm{C}_{2} \mathrm{~V}_{2} \mathrm{M}_{2}$ & $94.00 \mathrm{abc}$ & $92.00 \mathrm{ab}$ & $90.00 \mathrm{ab}$ & $87.00 \mathrm{abc}$ & $84.00 \mathrm{a}$ \\
\hline $\mathrm{C}_{2} \mathrm{~V}_{2} \mathrm{M}_{3}$ & $90.00 \mathrm{~b}-\mathrm{e}$ & $82.00 \mathrm{ef}$ & $62.00 \mathrm{e}$ & $48.00 \mathrm{e}$ & $40.00 \mathrm{c}$ \\
\hline $\mathrm{C}_{2} \mathrm{~V}_{2} \mathrm{M}_{4}$ & $80.00 \mathrm{gh}$ & $70.00 \mathrm{~g}$ & $40.00 \mathrm{fg}$ & $26.00 \mathrm{gh}$ & $18.00 \mathrm{~d}$ \\
\hline $\mathrm{C}_{2} \mathrm{~V}_{3} \mathrm{M}_{1}$ & $92.00 \mathrm{a}-\mathrm{e}$ & $86.00 \mathrm{~b}-\mathrm{e}$ & $84.00 \mathrm{bc}$ & $82.00 \mathrm{bc}$ & $80.00 \mathrm{a}$ \\
\hline $\mathrm{C}_{2} \mathrm{~V}_{3} \mathrm{M}_{2}$ & $92.00 \mathrm{a}-\mathrm{d}$ & $86.00 \mathrm{~b}-\mathrm{e}$ & $84.00 \mathrm{bc}$ & $82.00 \mathrm{bc}$ & $80.00 \mathrm{a}$ \\
\hline $\mathrm{C}_{2} \mathrm{~V}_{3} \mathrm{M}_{3}$ & $89.33 c-f$ & $67.00 \mathrm{~g}$ & $48.66 f$ & $34.00 \mathrm{fg}$ & $36.00 \mathrm{c}$ \\
\hline $\mathrm{C}_{2} \mathrm{~V}_{3} \mathrm{M}_{4}$ & $85.33 \mathrm{~d}-\mathrm{g}$ & $60.00 \mathrm{~g}$ & $34.66 \mathrm{gh}$ & $21.33 \mathrm{~h}$ & $12.00 \mathrm{de}$ \\
\hline CV (\%) & 4.95 & 6.69 & 7.23 & 8.20 & 7.32 \\
\hline
\end{tabular}

$\mathrm{CV}=$ Coefficient of variation, $\mathrm{C}_{1}=$ Polythene bag, $\mathrm{C}_{2}=$, Glass jar, $\mathrm{V}_{1}=\mathrm{AGS}-191, \mathrm{~V}_{2}=$ ASET-93.

$\mathrm{V}_{3}=$ Shohag, $\mathrm{SMC}=$ Seed moisture content, $\mathrm{M}_{1}=6 \% \mathrm{SMC}, \mathrm{M}_{2}=8 \% \mathrm{SMC}, \mathrm{M}_{3}=10 \%$ $\mathrm{SMC}, \mathrm{M}_{4}=12 \% \mathrm{SMC}$.

In a column, figures having similar letter(s) do not differ significantly. 
Table 4. Interaction of storage container, genotype and seed moisture content on germination index of soybean seed at different days after storage

\begin{tabular}{|c|c|c|c|c|}
\hline \multirow{2}{*}{$\begin{array}{c}\text { Container×genotype } \\
\times \text { initial SMC }\end{array}$} & \multicolumn{4}{|c|}{ Germination index at different days after storage } \\
\hline & 50 & 100 & 150 & 200 \\
\hline $\mathrm{C}_{1} \mathrm{~V}_{1} \mathrm{M}_{1}$ & $38.00 \mathrm{ab}$ & $34.00 \mathrm{a}$ & $28.00 \mathrm{ab}$ & $24.33 \mathrm{abc}$ \\
\hline $\mathrm{C}_{1} \mathrm{~V}_{1} \mathrm{M}_{2}$ & $38.00 \mathrm{ab}$ & $35.00 \mathrm{a}$ & $27.00 \mathrm{ab}$ & $23.66 \mathrm{abc}$ \\
\hline $\mathrm{C}_{1} \mathrm{~V}_{1} \mathrm{M}_{3}$ & $34.00 \mathrm{a}-\mathrm{e}$ & 26.00def & $18.00 \mathrm{de}$ & $12.00 \mathrm{de}$ \\
\hline $\mathrm{C}_{1} \mathrm{~V}_{1} \mathrm{M}_{4}$ & $34.00 \mathrm{a}-\mathrm{e}$ & $19.33 \mathrm{~h}$ & $9.0 \mathrm{gh}$ & $1.00 \mathrm{~h}$ \\
\hline $\mathrm{C}_{1} \mathrm{~V}_{2} \mathrm{M}_{1}$ & $38.00 \mathrm{ab}$ & $32.00 \mathrm{abc}$ & 27.00ab & $24.33 \mathrm{abc}$ \\
\hline $\mathrm{C}_{1} \mathrm{~V}_{2} \mathrm{M}_{2}$ & $37.00 \mathrm{abc}$ & $30.00 \mathrm{a}-\mathrm{d}$ & $26.00 \mathrm{ab}$ & $24.33 \mathrm{abc}$ \\
\hline $\mathrm{C}_{1} \mathrm{~V}_{2} \mathrm{M}_{3}$ & $32.00 \mathrm{c}-\mathrm{f}$ & $25.00 \mathrm{~d}-\mathrm{g}$ & $18.00 \mathrm{de}$ & $11.00 \mathrm{def}$ \\
\hline $\mathrm{C}_{1} \mathrm{~V}_{2} \mathrm{M}_{4}$ & $30.00 \mathrm{ef}$ & $20.00 \mathrm{gh}$ & $7.00 \mathrm{gh}$ & $1.37 \mathrm{~h}$ \\
\hline $\mathrm{C}_{1} \mathrm{~V}_{3} \mathrm{M}_{1}$ & 31.00def & $27.00 \mathrm{cde}$ & $24.00 \mathrm{abc}$ & $20.66 b c$ \\
\hline $\mathrm{C}_{1} \mathrm{~V}_{3} \mathrm{M}_{2}$ & $30.00 \mathrm{ef}$ & $27.00 \mathrm{cde}$ & $23.00 \mathrm{bcd}$ & $20.33 \mathrm{c}$ \\
\hline $\mathrm{C}_{1} \mathrm{~V}_{3} \mathrm{M}_{3}$ & $24.00 \mathrm{gh}$ & 18.00hi & 11.00fg & 8.00efg \\
\hline $\mathrm{C}_{1} \mathrm{~V}_{3} \mathrm{M}_{4}$ & $20.00 \mathrm{~h}$ & $12.00 \mathrm{j}$ & $5.00 \mathrm{~h}$ & $1.00 \mathrm{~h}$ \\
\hline $\mathrm{C}_{2} \mathrm{~V}_{1} \mathrm{M}_{1}$ & $39.00 \mathrm{a}$ & $35.00 \mathrm{a}$ & $30.00 \mathrm{a}$ & $26.33 a$ \\
\hline $\mathrm{C}_{2} \mathrm{~V}_{1} \mathrm{M}_{2}$ & $39.00 \mathrm{a}$ & $34.00 \mathrm{a}$ & $28.00 \mathrm{ab}$ & $25.55 \mathrm{ab}$ \\
\hline $\mathrm{C}_{2} \mathrm{~V}_{1} \mathrm{M}_{3}$ & $36.00 \mathrm{a}-\mathrm{d}$ & $30.00 \mathrm{a}-\mathrm{d}$ & $24.00 \mathrm{abc}$ & $14.33 \mathrm{~d}$ \\
\hline $\mathrm{C}_{2} \mathrm{~V}_{1} \mathrm{M}_{4}$ & $34.00 \mathrm{a}-\mathrm{e}$ & $22.00 \mathrm{e}-\mathrm{h}$ & $15.00 \mathrm{ef}$ & $4.40 \mathrm{gh}$ \\
\hline $\mathrm{C}_{2} \mathrm{~V}_{2} \mathrm{M}_{1}$ & $38.00 \mathrm{ab}$ & $33.00 \mathrm{ab}$ & $29.00 \mathrm{ab}$ & $25.66 \mathrm{ab}$ \\
\hline $\mathrm{C}_{2} \mathrm{~V}_{2} \mathrm{M}_{2}$ & $38.00 \mathrm{ab}$ & $32.00 \mathrm{abc}$ & $28.00 \mathrm{ab}$ & $26.00 \mathrm{a}$ \\
\hline $\mathrm{C}_{2} \mathrm{~V}_{2} \mathrm{M}_{3}$ & $33.00 \mathrm{~b}-\mathrm{e}$ & $26.00 \mathrm{def}$ & $20.00 \mathrm{cde}$ & $15.00 \mathrm{~d}$ \\
\hline $\mathrm{C}_{2} \mathrm{~V}_{2} \mathrm{M}_{4}$ & $31.00 \mathrm{def}$ & $21.00 f g h$ & $10.00 f g h$ & $5.10 \mathrm{gh}$ \\
\hline $\mathrm{C}_{2} \mathrm{~V}_{3} \mathrm{M}_{1}$ & $32.00 \mathrm{c}-\mathrm{f}$ & $28.00 \mathrm{bcd}$ & $25.00 \mathrm{abc}$ & $22.33 \mathrm{abc}$ \\
\hline $\mathrm{C}_{2} \mathrm{~V}_{3} \mathrm{M}_{2}$ & $32.00 \mathrm{c}-\mathrm{f}$ & $27.00 \mathrm{cde}$ & $24.00 \mathrm{abc}$ & $21.66 \mathrm{abc}$ \\
\hline $\mathrm{C}_{2} \mathrm{~V}_{3} \mathrm{M}_{3}$ & $28.00 \mathrm{fg}$ & $22.00 \mathrm{e}-\mathrm{h}$ & $15.00 \mathrm{ef}$ & $7.00 \mathrm{fg}$ \\
\hline $\mathrm{C}_{2} \mathrm{~V}_{3} \mathrm{M}_{4}$ & $22.00 \mathrm{~h}$ & $14.00 \mathrm{ij}$ & $8.00 \mathrm{gh}$ & $3.66 \mathrm{gh}$ \\
\hline $\mathrm{CV}(\%)$ & 8.41 & 6.48 & 5.75 & 7.32 \\
\hline
\end{tabular}

$\mathrm{CV}=$ Coefficient of variation, $\mathrm{C}_{1}=$ Polythene bag, $\mathrm{C}_{2}=$, Glass jar, $\mathrm{V}_{1}=\mathrm{AGS}-191$.

$\mathrm{V}_{2}=$ ASET $-93, \mathrm{~V}_{3}=$ Shohag, $\mathrm{SMC}=$ Seed moisture content, $\mathrm{M}_{1}=6 \% \mathrm{SMC}, \mathrm{M}_{2}=8 \%$ SMC, $\mathrm{M}_{3}=10 \% \mathrm{SMC}, \mathrm{M}_{4}=12 \% \mathrm{SMCIn}$ a column, figures having similar letter(s) do not differ significantly. 


\section{Germination index}

The interaction of storage container, genotypes and initial seed moisture content on germination index was statistically significant at each of the observation dates during the storage period in 2013. The highest germination index for AGS 191 in glass jar with 6\% initial MC were 39.00, 35.00, 30.00 and 26.33, respectively at 50,100,150 and 200 DAS and it was statistically at par to those of AGS 191 in glass jar with 8\% initial MC, ASET 93 in polythene bag with at $6 \%$ and $8 \%$ SMC, Shohag in glass jar with 6 or $8 \%$ initial MC. Those values for Shohag in polythene bag with $12 \%$ initial MC were 20.00, 12.00, 5.00 and 1.00 and it was statistically identical to Shohag in glass jar with $12 \%$ initial MC, respectively (Table 4). These results are conformity of those reported by Balesevic-Tubic et al., 2005. Agha et al., 2004 reported that when seed could be packaged in moisture proof containers, the relative humidity of the air around the seed remains low, then the seed equilibrium moisture remains low and the seed maintains its viability and vigour for a longer time.

\section{Conclusion}

The study conclude that seeds of AGS 191, ASET 93 and Shohag could be stored in polythene bag or glass jar at 6 to $8 \%$ initial seed moisture content safely for maintaining high seed quality. However, seed with high moisture content should never be stored in air tight container.

\section{References}

Agha, S. K., Z. H. Malik, M. Hatam and G. H. Jamro. 2004. Emergence of healthy seedlings of soybeans as influenced by seed storage containers. Pakistan J. Biol. Sci. 7(1): 42-44.

Ali, M. R., M. M. Rahman, M. A. wadud, A. H. F.Fahim and M. S. Nahar. 2018. Effect of seed moisture content and storage container on seed viability and vigor soybean. Bangladesh Agron. J. 21(1):131-141.

Alam, M. M. and M. M. Rahman. 2005. Effect of seed moisture content and thickness of polythene bag on germination and vigour on soybean seed. Bangladesh Seed Sci. Technol. 9(1\&2): 33-38.

Arunnandhy, V. and Senanayaka, Y.D.A. 1991. Changes in viability, vigour and chemical composition of soybean seeds stored under the humid tropical conditions. Legume Research. 14(3):135-140.

Balesevic-Tubic, M. Tatic, V. DorDevic, Z. Nikolic, J. Subic and V. Dukic. 2011. Changes in soybean seeds as affected by accelerated and natural ageing. Romanian Biotechnological Letters. 16(6):6740-6747.

Balesevic-Tubic, M. Tatic, V. DorDevic, Z. Nikolic, J. Subic and V. Dukic. 2011. Changes in soybean seeds as affected by accelerated and natural ageing. Romanian Biotechnological Letters. 16(6):6740-6747. 
Kong, F., S. K. C. Chang, Z. Liu and L. A. Wilson. 2008. Change of soybean quality during storage as related to soyamilk and tofu making. J. Food Sci. 73(3): 134-144.

Poonam S., N. Tewari, P. K. Katiyar, T. Das and C.P. Vaish. 2001. Storability of soybean (Glycine max Merr.) seeds in different containers under ambient conditions. Progres. Agric. 1(1): 90-93.

Rahman, M. M., B. Hagidok, M. M. Masood and M. N. Islam. 2010. Effect of storage container and relative humidity on the quality of wheat seed. Bangladesh J. Seed Sci. Technol. 14(1\&2): 89-94.

Rahman, L. 2001. Soybean: Production Food Uses Technology (in Bangla). BAU-USDA Soybean Project, Dept. Genetics and Plant Breeding. Bangladesh Agril. Univ., Mymensingh, pp. 1-24.

Tatic M, S. Balesevic-Tubic, V. Dordevic, V. Miklic, M. Vujakovic and V. Dukic. 2012. Vigor of sunflower and soybean ageing seed. Helia. 25(56):119-126.

Tatipata, A. 2009. Effect of seed moisture content packaging and storage period on microchondria inner membrane of soybean seed. J. Agric. Technol. 5(1): 51-54. 